\title{
Mode identification in the pulsating subdwarf Balloon 090100001 by means of the spectrophotometric method
}

\author{
A. Baran, ${ }^{1,2}$ A. Pigulski, ${ }^{3}$ S. J. O' Toole $^{4}$ \\ ${ }^{1}$ Mt. Suhora Observatory, Poland \\ 2 Torun Centre for Astronomy, Poland \\ ${ }^{3}$ Astronomical Institute, University of Wrocław, Poland \\ ${ }^{4}$ University of Sydney, Australia
}

\begin{abstract}
We present the first successful application of the spectrophotometric method of mode identification to a pulsating subdwarf B star, Balloon 090100001. We confirm that the dominant mode is radial and that the observed triplet can be interpreted in terms of a rotationally split dipole mode.
\end{abstract}

\section{The data and results}

Balloon 090100001 (hereafter Bal09), a recently discovered pulsating subdwarf, appeared to be extremely interesting because both $\mathrm{p}$ and $\mathrm{g}$ modes were revealed in its frequency spectrum. In addition, two rotationally split multiplets, a triplet and a quintuplet, were detected.

The star was observed during two photometric campaigns in 2004 (Baran et al. 2005, Oreiro et al. 2005) and 2005. Multicolour BVRI photometry was obtained. Simultaneously with the photometric observations, spectroscopic observations were carried out by Telting \& $\emptyset$ stensen (2006). Six modes with the largest amplitudes were detected in the radial velocities. Using the photometric and spectroscopic observations, we applied the method elaborated by Daszyńska-Daszkiewicz et al. (2003) to Bal09. A grid of model atmospheres (Heber et al. 2000) for sdB stars was calculated for this purpose. Since six modes were detected in radial velocities, only these modes could be identified by means of the full version of the method.

The results of the application of the spectrophotometric method to Bal09 are shown in Fig. 1. From the data available to us, we were not able to unambiguously identify the $\ell$ values of any of the detected modes on the basis of the photometry alone. Including spectroscopy, we get unambiguous discrimination of $\ell$ for the two modes with the largest amplitudes, $f_{1}$ and $\mathrm{f}_{2}$, while for the others, two or three values of $\ell$ are equally possible. From the frequency pattern, we have already suggested (Baran et al. 2005) that the dominant mode in Bal09 is radial and the triplet represents the rotationally split $\ell=1$ mode. This is now confirmed by this work, as the strongest triplet component, $f_{2}$, has a convincingly identified value of $\ell$ equal to 1 , and for the other two, $f_{3}$ and $f_{4}, \ell=1$ is one of the possibilities.

Acknowledgments. This work was supported by the grant 1 P03D 01329.

\section{References}

Baran A., Pigulski A., Kozieł D., et al., 2005, MNRAS, 360, 737

Daszyńska-Daszkiewicz J., Dziembowski W. A., Pamyatnykh A. A., 2003, A\&A, 407, 999

Heber U., Reid I. N., Werner K., 2000, A\&A, 363, 198

Oreiro R., Pérez Hernández F., Ulla A., et al., 2005, A\&A, 438, 257

Telting J. H., Østensen R., 2006, A\&A, 450, 1149 


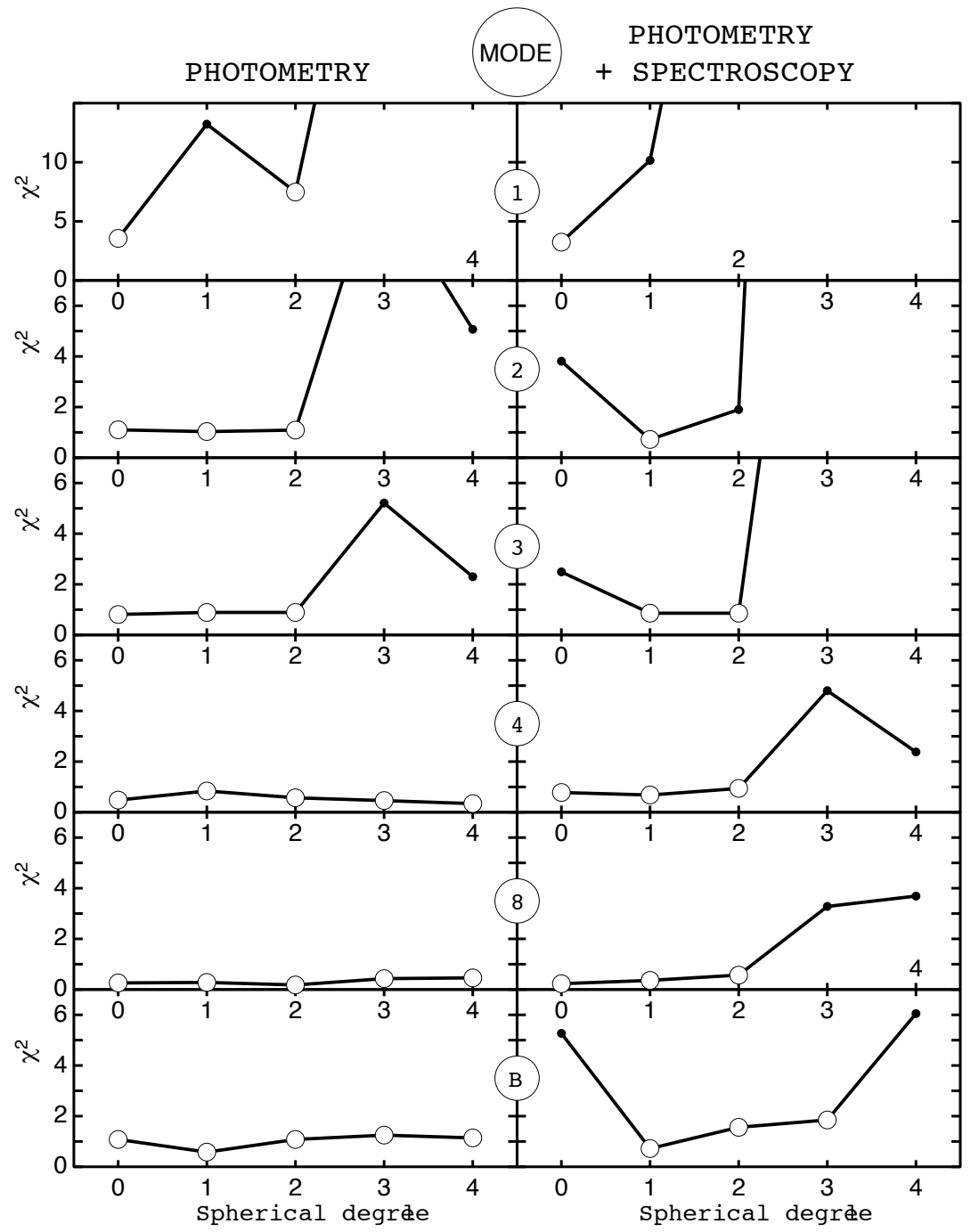

Figure 1: Discrimination of the spherical degree $\ell$ for the six strongest modes in Bal09 by means of the spectrophotometric method. The panels show reduced $\chi^{2}$ for five different spherical degrees $\ell$, ranging from 0 to 4 , for six modes detected in spectroscopy. Open circles indicate the result of the discrimination, i.e., the possible values of $\ell$. 